\title{
Digital Modulator and Demodulator IC for RFID Tag Employing DSSS and Barker Code
}

\author{
M. S. Amin $\star^{1}$, M. B. I. Reaz ${ }^{2}$, J. Jalii ${ }^{3}$, L. F. Rahman ${ }^{4}$ \\ 1,2, 34 Department of Electrical, Electronic and Systems Engineering \\ Universiti Kebangsaan Malaysia \\ 43600 UKM, Bangi, Selangor, Malaysia \\ *amin.syedul@gmail.com
}

\begin{abstract}
Radio frequency identification (RFID) is lagging behind because of vendor specific solutions and expensive implementation cost. In particular, the reader is the most expensive part. A WiFi compatible tag was proposed to use the WNIC as an RFID reader. However, no specific modulator or demodulator was suggested. This paper analyzes the various IEEE 802.11 standards and their modulation and coding techniques keeping the desired properties of an RFID system in consideration. After the analysis, a digital modulator and demodulator for RFID tag in IEEE 802.11 protocol employing Direct Sequence Spread-Spectrum (DSSS) and coding is proposed. A MOD-11 synchronous counter is designed for the 11-bit encoder which generates the desired Barker code. Data are multiplied with this Barker code to modulate the data, and the received data are multiplied with the Barker code to demodulate them. The proposed modulator and demodulator are implemented in $0.18 \mu \mathrm{m}$ CMOS technology. The simulation results show that 1 bit is spread to 11 bits by the modulator and 11-bit received data are demodulated to 1 bit correctly. The proposed design is simple, resistant to multipath fading and interference and offers the highest distance with the lowest BER for an RFID tag.
\end{abstract}

Keywords: Barker code, IEEE 802.11, modulator, RFID, WLAN.

\section{Introduction}

RFID is a very familiar and emerging technology which is being used everywhere nowadays. It offers realistic benefits nearly to everyone. RFID is the utilization of radio waves to transfer data between a reader and a tag attached to an object for the purpose of identification and tracking. RFID consists of two fundamental components: a tag or transponder and a transceiver or reader. The tag is an integrated circuit with an antenna which keeps the data and responds to the request of the reader. The reader reads the data through wireless communication. The tag uses an Electronic Product Code (EPC), which is a unique number attached inside for identification.

The IEEE 802.11 standard in the $2.4 \mathrm{GHz}$ ISM band has emerged as a prevailing technology for wireless access. It provides data rates from one megabit per second (Mbps) to several Mbps. The IEEE 802.11 protocol-based wireless communication is robust, resistant to interference and has more range and a lower bit error rate
(BER) [1]. Due to the requirement of a global unique address structure for object-to-object communications, the IPV6 protocol will be used in coming days instead of the IPv4 one [2]. In contrast to WLAN, an RFID network is less secure, but more expensive. In an RFID system, the reader is the most expensive part [3]. To get rid of the vendor specific solutions and excessive implementation cost, an innovative RFID tagging system using WiFi is proposed where the built-in cheap Wireless Network Interface Card (WNIC) of a laptop or PC can be utilized as a reader [4]. The EPC would be mapped in the IPv6 protocol that is only 128 bits. However, the paper does not highlight the modulation and demodulation technique for the tag as IEEE 802.11 has different standards.

Institute of Electrical and Electronics Engineers, Inc (IEEE) released the 802.11 standards in 2.4GHz band in 1997 for wireless LANs, defining 1 and $2 \mathrm{Mbps}$ data rate [5]. It was ratified in September 1999 for higher rate to $5.5 \mathrm{Mbps}$ and 11 
Mbps as IEEE 802.11b. The hunt for higher speed brought the $802.11 \mathrm{~g}$ in 2003 for $54 \mathrm{Mbps}$. IEEE $802.11 \mathrm{n}$ reached the speed of $300 \mathrm{Mbps}$ recently. To attain the higher data rate, changes were basically made only to the physical layer [6]. The higher data rate needs complex circuitry at the physical layer which increases cost as well as power consumption. Whenever the BER increases because of speed, distance, noise or interference; all the IEEE 802.11 standards gradually fall to the basic data rate of 802.11 which are 1 and 2 Mbps [6].

This paper discusses the different IEEE 802.11 standards and proposes 1 Mbps data rate in Direct Sequence Spread Spectrum (DSSS) technique employing the Barker code for the digital modulator and demodulator of the RFID tag. Higher range, anti-interference, anti multipath fading and lower BER were considered while selecting modulation and demodulation techniques. The modulation and demodulation techniques will not only be applicable for the IEEE 802.11 protocol compatible tag, but they can also be used in other systems as they can overcome the drawbacks of the conventional tag problems like multipath, interference, security, short distance, BER, etc. The proposed modulator and demodulator are designed using the $0.18 \mu \mathrm{m}$ CMOS technology.

The rest of the paper is organized as follows. The "Design considerations" section shows the advantages of using $1 \mathrm{Mbps}$ data rate in DSSS employing the Barker code. The "Barker code sequence design" section describes the details of the generation of the Barker code, the modulator and demodulator design by using the Barker code. "Simulation results" and "Discussion" section covers the simulation result and related discussion ann finally the paper is concluded.

\section{Design considerations}

IEEE 802.11 utilizes the spread-spectrum communication technique in which the energy used in transmitting the signal is spread over a wider bandwidth which appears as noise. There are mainly two types of spread-spectrum techniques: Frequency Hopping Spread-Spectrum (FHSS) and DSSS. In FHSS, the carrier frequency hops from channel to channel in a pre-arranged sequence. The major drawbacks of the FHSS are limited data rate, poor range resolution and interference to other systems, requirement of positive SNR and lesser efficiency in noncoherent modulation [7].

On the contrary, in DSSS, a pseudorandom code known as chipping code is directly applied to the data for spreading before the carrier modulation [8]. The RF carrier with the pseudorandom digital code sequence consisting of $M$ chips accomplished by phase shift-keying, which is independent of the data symbol. As the chip rate is larger than the symbol rate, the resulting bandwidth is larger than the information signal bandwidth. It produces transmitted signals with low spectral densities and provides tolerances to multipath interference. Thus, the DSSS signal has the characteristics of pseudorandomness and correlation processing, which leads to many advantages, such as antinoise, anti-interference, anti-multipath fading, low power spectrum density, high secrecy, multiaccess flexibility and high precision measurements [9]. Considering these properties, DSSS is more suitable to implement in RFID.

DSSS resolves the multipath components and attenuates those paths with delays in excess of one chip duration from the in-phase autocorrelation peak [10]. The attenuation depends on the sidelobe values of the aperiodic autocorrelation function as these sidelobes show intersymbol interference in a dispersive channel. An autocorrelation function with zero sidelobe values is desirable, but it does not exist in the family of binary spreading sequences [10]. The Barker sequence is close to this property which possesses good aperiodic correlation. Let $\left\{a_{n}\right\}$ denote a real-valued binary sequence of length $N$, where the sequence elements $a_{n} \in\{-1,+1: n=0,1, \ldots . N-1\}$ are referred to as sequence chips. Then, the aperiodic autocorrelation function $\left\{a_{n}\right\}$, denoted $C_{a}(\tau)$ for an integer chip delay $\tau$, is given by (1).

$$
C_{a}(\tau)=\sum_{n=0}^{N-\tau-1} a_{n} a_{n+r}
$$

Ronald $\mathrm{H}$. Barker considered the problem of synthesizing real-valued binary sequences with aperiodic autocorrelation function sidelobes bounded by $\left|C_{a}(\tau)\right| \leq 1$. Binary sequences that 
satisfy these bounds are known as Barker sequences. Only Barker codes of lengths $2,3,4$, $5,7,11$ and 13 exist [11]. It is conjectured that Barker codes no longer exist. The list of known Barker codes is given in Table 1.

\begin{tabular}{|c|c|}
\hline length & code \\
\hline 2 & 10,11 \\
\hline 3 & 110 \\
\hline 4 & 1101,1110 \\
\hline 5 & 11101 \\
\hline 7 & 1110010 \\
\hline 11 & 11100010010 \\
\hline 13 & 1111100110101 \\
\hline
\end{tabular}

Table 1. List of known Barker codes.

The Barker code produces a single peak and uniformly low sidelobes when correlated against time-shifted versions of itself and thereby it rejects multipath $[12,13]$. This simply means that due to the nonrepetitive behavior of the code, a matched filter correlator can easily identify the location of a Barker code in a sequence of bits [14]. If one or more chips in the bit are lost during transmission, statistical techniques embedded in the receiver can recover the original data without retransmission. IEEE 802.11 utilizes the 11-bit Barker code. The value of the autocorrelation function for the 11-bit Barker code is 1, -1 , or 0 at all offsets except zero, where it is 11 . The autocorrelation function of the 11-bit Barker code is shown in Figure 1.

In IEEE 802.11, the data to be transmitted are modulated with the 11-bit Barker code and thereby the data are spread over a wide bandwidth. On the other hand, data to be demodulated are again multiplied with the same 11-bit Barker code which despreads the data and the data are recovered. The block diagram of the proposed modulator and demodulator utilizing the Barker code is shown in Figure 2.

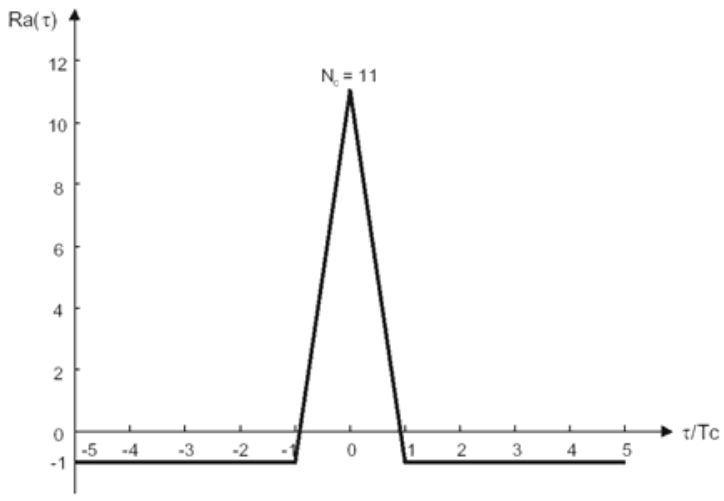

Figure 1. Autocorrelation function of the 11-bit Barker. code.

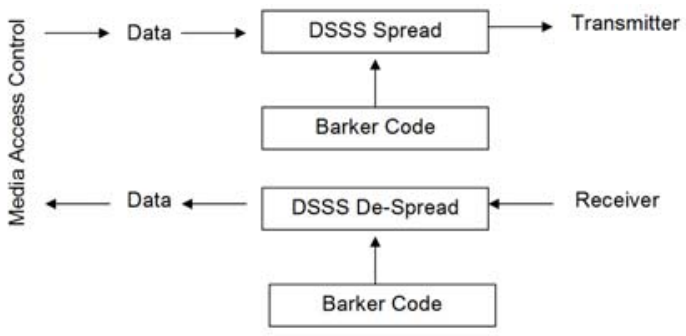

Figure 2. Proposed modulator and demodulator for RFID tag.

\section{Barker code, modulator and demodulator design}

The heart of the modulator and demodulator is the 11-bit Barker code which is a sequence of 11100010010. A code generator can be designed by using different approaches. Normally, a Barker code generator uses preloaded register as per the sequence of the code. But in this paper, the novelty of the code generator is the utilization of four flips only. A very simple and systematic approach is followed for designing the Barker code. At first, an MOD-11 synchronous counter is designed which counts from 0 to 10 . The counter uses only four flip flops. This counter is fed in the 
11-bit encoder and the output of the encoder is the 11-bit Barker code. The block diagram for the Barker code is shown in Figure 3.

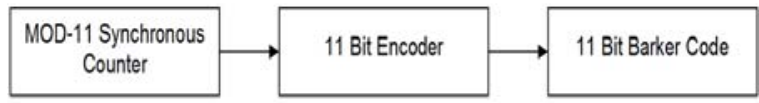

Figure 3. Block diagram of the 11-bit Barker code generator.

\subsection{MOD-11 synchronous counter}

A synchronous binary counter counts from 0 to $2^{N}$ 1 , where $N$ is the number of bits/flip-flops in the counter and thus it will have $n=2^{N}$ states where $n$ is the number of states [15]. For the Barker code, a counter is needed where it will have 11 distinct states. Hence, by using $n=2^{N}$, the minimum number of flip flops is found to be 4 . With the four flip-flops the number of states will be 16 as $n=2^{N}$. However, the Barker code generator requires 11 distinct states. Thus, after counting 11 states, the rest of the states need to be ignored. This type of counter is known as MOD counter and requires additional combinational logic. For the 11-bit Barker code, a MOD-11 synchronous counter is required. It is assumed that the state transition from one state to the next state takes place during a clock transition. The state diagram for the 11 states $(0000$ to 1010) is shown in Figure 4. The state diagram is then converted to a state table. The $D$ flip-flop of the CEDEC standard library in Mentor Graphics is considered for the counter. An excitation table for the $D$ flip-flop is created from the state table as shown in Table 2. The excitation table is then simplified and minimized by Karnaugh map.

\subsection{1-bit encoder}

For the 11-bit encoder, the Barker code 10110111000 is considered as the left most bit would be output first in time. The sequence is written in a table sequentially with a count from binary 0 to 10 (0000 to 1010). The code sequence is then simplified and minimized by Karnaugh map.

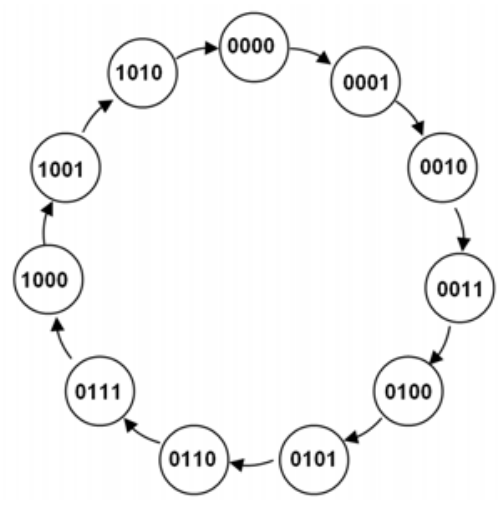

Figure 4. State diagram for the MOD-11 synchronous counter.

\begin{tabular}{|c|c|c|c|c|c|c|c|}
\hline A3 & A2 & A1 & A0 & D3 & D2 & D1 & D0 \\
\hline 0 & 0 & 0 & 0 & 0 & 0 & 0 & 1 \\
\hline 0 & 0 & 0 & 1 & 0 & 0 & 1 & 0 \\
\hline 0 & 0 & 1 & 0 & 0 & 0 & 1 & 1 \\
\hline 0 & 0 & 1 & 1 & 0 & 1 & 0 & 0 \\
\hline 0 & 1 & 0 & 0 & 0 & 1 & 0 & 1 \\
\hline 0 & 1 & 0 & 1 & 0 & 1 & 1 & 0 \\
\hline 0 & 1 & 1 & 0 & 0 & 1 & 1 & 1 \\
\hline 0 & 1 & 1 & 1 & 1 & 0 & 0 & 0 \\
\hline 1 & 0 & 0 & 0 & 1 & 0 & 0 & 1 \\
\hline 1 & 0 & 0 & 1 & 1 & 0 & 1 & 0 \\
\hline 1 & 0 & 1 & 0 & 0 & 0 & 0 & 0 \\
\hline
\end{tabular}

Table 2. State and excitation table for D flop-flops.

\subsection{1-bit Barker code}

The 11-bit encoder needs the signals from the 11bit counter. The connections are drawn according to the minimized Karnaugh map of the 11-bit Barker encoder. When the counter is activated by clocking, the 11-bit encoder outputs the Barker code as per the sequence which is 10110111000 and then the process is repeated.

\subsection{Modulator and demodulator}

According to the principle of DSSS, for modulation, the data need to be multiplied with the 11-bit Barker code. An XOR gate is used to multiply the data with the 11-bit Barker code. In one input of the XOR gate, 
the data to be transmitted are connected and, at the other input, the Barker code output are connected. As per the principle of XOR gate, for data representing $1 \mathrm{bit}$, the Barker code will have a reverse phase;nevertheless, for 0 bit, it will remain same. For demodulation, the received data are again multiplied with the same Barker code. An XOR gate is used for the demodulation. At one input of the XOR gate, the received data are connected and, at the other input, the Barker code output is connected. When these data are multiplied with the Barker code, the data are recovered. As such, the output of the XOR gate provides the demodulated data. The schematic diagram of the modulator and demodulator with the 11-bit Barker code in $0.18 \mu \mathrm{m}$ CMOS process technology using CEDEC standard library is shown in Figure 5. A part of the layout design is shown in Figure 6.

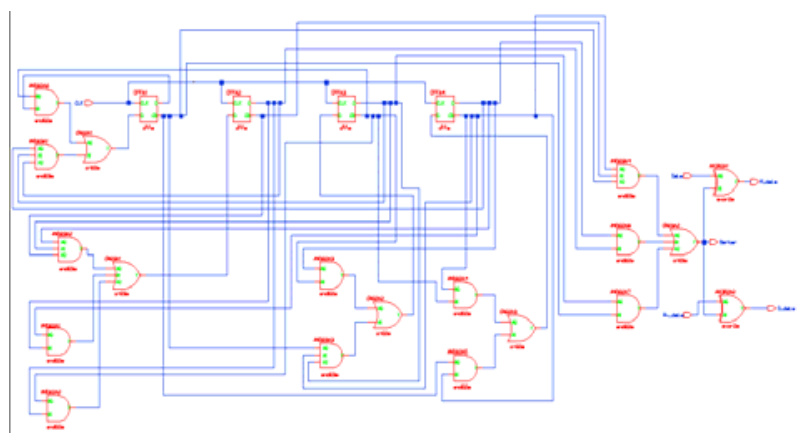

Figure 5. Schematic diagram of proposed modulator and demodulator.

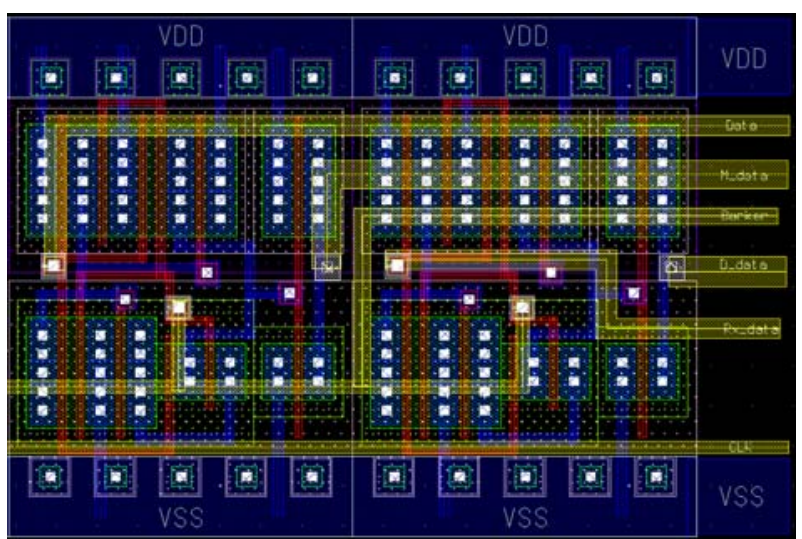

Figure 6. A part of the layout of the modulator and demodulator.

\section{Results and discussion}

The simulations of the designed RFID tag modulator and demodulator are carried out utilizing an ELDONET simulator. DC $1.8 \mathrm{~V}$ is used as power supply. The data rate of the modulator and demodulator is 1 Mbps which means the period of 1 bit is $1 \mu \mathrm{s}$. These data are modulated with the 11bit Barker code. As such, the period of 1 bit of the Barker code is $1 / 11$ of a $\mu$ s which is 90.909090 ns. Thereby, the clock pulse period is $90.909090 \mathrm{~ns}$ and the width is $45.454545 \mathrm{~ns}$. The initial value of the clock is set to 0 and $1.8 \mathrm{v}$ pulse is used. The rise time and fall time for the clock are used as 0.1 ns. The simulation result in Figure 7 shows that the counter can count from 0 to 11 and a correct 11-bit Barker code is generated from the encoder which is a sequence of 10110111000 . Instead of using 11 flip-flops by preloading for the 11-bit Barker code sequence, the four flip-flops with the combinational logic show the correct pattern of the 11-bit Barker code. The reduction of the number of flip-flops is the novelty of the module. For the data to be modulated, a pattern of 101 is used with 1 ns period. The data 101 are modulated with the Barker code. For the 1 bit, the Barker code is inverted, and for the 0 bit, the Barker code remains unchanged. As such, the modulated output data for $101 \quad$ become 101101110000100100011110110111000 . These results show the correct functionality of the modulator.

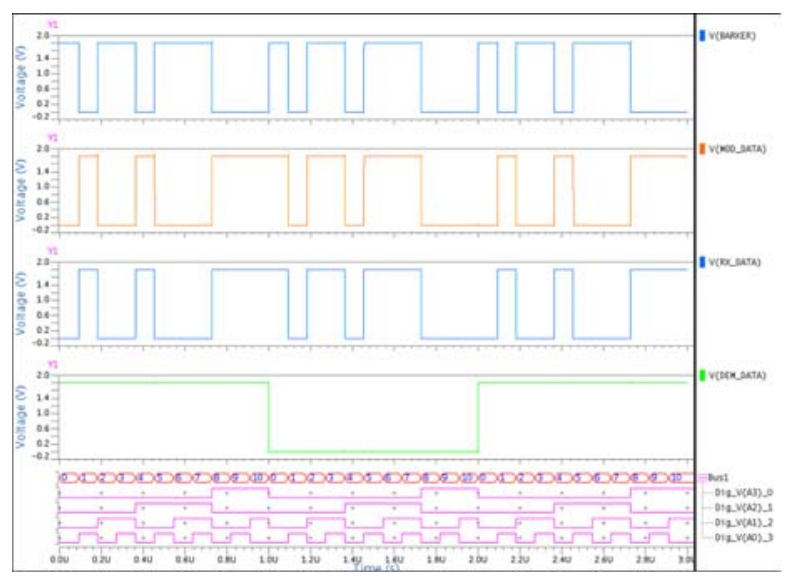

Figure 7. Simulation result of the proposed modulator and demodulator. 
For the simulation of the demodulator, a pattern of 101101110000100100011110110111000 is used as the received data. The period of the data pattern is considered $90.909090 \mathrm{~ns}$ as the data are a multiplication of 11-bit Barker code. These data are multiplied again with the 11-bit Barker code. As such, the data are recovered to a pattern of 101 by canceling out the Barker code. Thereby, the simulation result in Figure 7 shows the correct functionality of the demodulator.

\section{Conclusion}

Both RFID and WLAN are popular and widely used technologies. RFID suffers from vendor specifications and huge implementation cost. It is also susceptible to noise, interference and multipath fading. On the other hand, IEEE 802.11 has a standard with different variations for achieving higher data rate with a backward compatibility to 1 Mbps. The proposed RFID tag only needs to transfer the 128-bit EPC mapped IPv6 address. As such, the designed digital modulator and demodulator using the 11-bit Barker code can easily pass the 128-bit data of the tag address. The design works towards the readerless RFID system by implementing a simple modulator and demodulator using the Barker code. Besides being used in the IEEE 802.11 compatible protocol, the proposed modulator and demodulator can also be used in other RFID systems to get rid of the fading, multi-path, interference, etc.

\section{References}

[1] W. Jiang and M. A. Yan, "Bit Error Rate Analysis of Wi-Fi and Bluetooth under the Interference of $2.45 \mathrm{GHz}$ RFID," The Journal of China Universities of Posts and Telecommunications, vol. 14, no. 4, pp. 89-93, 2007.

[2] G. M. Lee, J. K. Choi and T. Chung, "Address Structure for Supporting Ubiquitous Networking Using IPv6," in Proceeding of 10th International Conference on Advanced Communication Technology, Gangwon-Do, South Korea, 2008, pp. 1088-1090.

[3] L. F. Rahman, M. B. I. Reaz, M. A. M. Ali and M. Kamada, "Design of an EEPROM in RFID Tag: Employing Mapped EPC and IPv6 Address," in IEEE Asia Pacific Conference on Circuits and Systems APCCAS 2010, Kuala Lumpur, Malaysia, 2010, pp. 168-171.

[4] L. F. Rahman, M. B. I. Reaz, M. A. M. Ali, M. Marufuzzaman and M. R. Alam, "Beyond the WIFI: Introducing RFID System Using IPv6," in ITU-T 2010 Conference on Innovations for Future Networks and Services, Kaleidoscope: Beyond the Internet?, Pune, India, 2010, pp. 1-4.

[5] General Information: 802.11. [Online]. Available: http://www.ieee802.org/11/main.html

[6] J. Mikulka and S. Hanus, "CCK and Barker Coding Implementation in IEEE 802.11b Standard," in 17th International Conference on Radioelektronika, Brno, Czech Republic, 2007, pp. 1-4.

[7] G. Spasov and B. Ribov, "Spread Spectrum for Wireless Systems Using PIC Based Micro-Controllers as a Spreader," in 11th International Scientific Conference of the Romanian-German University of Sibiu, Sibiu, Romania, 2002, pp. 89-94.

[8] D. Terra, N. Kumar, N. Lourenco, L. N. Alves and R. L. Aguiar, "Design, development and performance analysis of DSSS-based transceiver for VLC," in International Conference on Computer as a Tool (EUROCON), Lisbon, Portugal, 2011, pp. $1-4$.

[9] Y. Zhan, Z. Cao and J. Lu, "Spread-Spectrum Sequence Estimation for DSSS Signal in Noncooperative Communication Systems," IEEE Proceedings on Communications, vol. 152, no. 4, pp. 476-480, 2005.

[10] T. O'Farrel, "Design and Evaluation of a High Data Rate Optical Wireless System for the Diffuse Indoor Channel Using Barker Spreading Codes and Rake Reception [Optical wireless communications]," IET Communications, vol. 2, no. 1, pp. 35-44, 2008. 
[11] R. H. Barker, "Group Synchronizing of Binary Digital Sequences, Communication Theory," Butterworth, London: W. Jackson, 1953, pp. 273-287.

[12] H. S. Kim and J. K. Lee, "Optimal PN Codes in a DS/SS Wireless LAN System," in Proceeding of 1994 IEEE International Symposium on Information Theory, Trondheim, Norway, 1994, pp. 226.

[13] S. König, M. Schmidt and C. Hoene, "Precise time of flight measurements in IEEE 802.11 networks by crosscorrelating the sampled signal with a continuous Barker code," in IEEE 7th International Conference on Mobile Adhoc and Sensor Systems (MASS 2010), CA, USA, 2010, pp. 642-649.

[14] W. Jin, Y. Ruiqing, L. Huaping, Z. Ru, "UWB Signaling for $\mathrm{CR}$ and Coherent Receivers and Localization with Weak Received Signals," in IEEE Conference on Ultra-Wideband (ICUWB 2011), 2011, pp. 322-326.

[15] A. Saha and N. Manna, "Digital Principles \& Logic Design," Infinity Science Press LLC, 2007 